\title{
Rapid Fabrication of Nanoporous Gold as a Suitable Platform for the Direct Electron Transfer-type Bioelectrocatalysis of Bilirubin Oxidase
}

\author{
Masahiro MIYATA, Kenji KANO, Osamu SHIRAI, and Yuki KITAZUMI* \\ Division of Applied Life Sciences, Graduate School of Agriculture, Kyoto University, Sakyo, Kyoto 606-8502, Japan \\ *Corresponding author: kitazumi.yuki.7u@kyoto-u.ac.jp
}

\section{ABSTRACT}

Nanoporous gold fabricated via anodization in a buffer solution comprising potassium chloride served as an effective scaffold for the direct electron transfer-type bioelectrocatalysis of bilirubin oxidase from Myrothecium verrucaria (BOD). BOD adsorbed on a porous gold electrode showed an increased population of effective orientations required for direct electron transfer reactions. The catalytic current of BOD reached an oxygen-transfer-limited value at the nanoporous gold electrodes despite neutral conditions. license agreement.

Keywords : Direct Electron Transfer, Bilirubin Oxidase, Nanoporous Gold, Anodization

\section{Introduction}

Direct electron transfer (DET)-type bioelectrocatalysis is a reaction, in which electrons can directly interact with enzymes and electrodes. ${ }^{1}$ Bilirubin oxidase (BOD) belongs to a family of multicopper oxidases, which catalyze the reduction of oxygen $\left(\mathrm{O}_{2}\right)$ to water. ${ }^{2}$ Because BOD is reported to have high activity in DET-type catalytic reaction under neutral conditions, ${ }^{3}$ it is considered as a promising catalyst for biocathode in biosensors, biofuel cells, bioreactors, and biosupercapacitors. ${ }^{2}$ BOD receives four electrons at the $\mathrm{T} 2 / 3$ cluster from $\mathrm{O}_{2}$ and directly transfers them to the electrode from the T1 site. ${ }^{2}$ The DET-type catalytic activity can be improved by accelerating the electron transfer kinetics, which is achieved by shortening the distance between the T1 site of the enzyme and the electrode surface. An improved catalytic activity can also be achieved by increasing the amount of BOD adsorbed on the surface.

In this regard, porous electrodes with large surface areas are attractive scaffolds for the DET-type bioelectrocatalysis. ${ }^{4}$ Porous structures are convenient and essential for increasing the amount of immobilized BOD per projected area. Additionally, their curvature effect can facilitate the interfacial electron transfer kinetics. ${ }^{5}$ Porous gold materials are inert electrodes having controllable pore structures. ${ }^{6}$ In recent years, the fabrication of porous gold electrodes via the anodization of gold electrodes in solutions containing a reductant has been actively investigated, owing to the ease of this method. ${ }^{7}$ Although, the nanoporous gold prepared by the anodization in oxalic acid or glucose solutions serves as an effective scaffold for the DET-type bioelectrocatalysis of BOD, it also requires a long electrolysis time for fabrication. ${ }^{8,9}$ This study reveals that the anodization of gold electrodes in a chloride ion-containing solution can provide an effective scaffold for the DET-type bioelectrocatalysis of BOD in a short period. The orientation of BOD at the nanoporous gold electrode has been discussed based on the kinetics of the bioelectrocatalysis.

\section{Experimental}

BOD (EC 1.3.3.5), derived from Myrothecium verrucaria, was purchased from Amano Enzyme Inc. (Japan) and used without further purification. $\mathrm{Au}$ disk electrodes with a diameter of $3.0 \mathrm{~mm}$ were purchased from BAS Inc. (Japan). All the chemicals used in this study were of analytical grade and purchased from Wako Pure Chemical Ind. Ltd. (Japan). All the solutions were prepared with ultrapure water $\left(>18.2 \mathrm{M} \Omega \mathrm{cm}^{-1}\right)$.

Electrochemical measurements were performed on an electrochemical analyzer (ALS 611B or 714C, BAS Inc., Japan). Steady-state voltammetric measurements were conducted with rotating disk electrodes (RRDE-2, BAS Inc., Japan) at a rotating speed of $4000 \mathrm{rpm}$. A platinum-deposited titanium mesh was used as the counter electrode. All the potentials in this study are in reference to an $\mathrm{Ag}|\mathrm{AgCl}| \mathrm{sat} . \mathrm{KCl}$ electrode. All the measurements were obtained at $25.0 \pm 0.2^{\circ} \mathrm{C}$ using a water-jacketed cell.

BOD-immobilized nanoporous $\mathrm{Au}$ electrodes were prepared as follows (details are given in SI). The Au electrodes were polished with alumina slurry ( $1 \mu \mathrm{m}$ and $0.05 \mu \mathrm{m}$ successively) and then rinsed with water. The Au electrodes were anodized at $1.19 \mathrm{~V}$ in a "symmetrical configuration" of a counter electrode (Fig. 1). To immobilize BOD, a $40 \mu \mathrm{L}\left(\mathrm{L}=\mathrm{dm}^{3}\right)$ of $50 \mathrm{mg} \mathrm{mL}^{-1}$ BOD solution was dropped and contacted on the electrode surface for $1 \mathrm{~h}$ at $4{ }^{\circ} \mathrm{C}$ under water-saturated conditions. The excess enzyme was washed away from the electrode surface with a buffer solution before the electrochemical measurements. The surface structures of the anodized $\mathrm{Au}$ electrodes were observed by a field emission scanning electron microscope (FE-SEM) (SU8000, Hitachi High-Technologies, Japan) at an acceleration voltage of $10.0 \mathrm{kV}$.

\section{Results and Discussion}

We found that an asymmetrical configuration of a counter electrode to $\mathrm{Au}$ electrode was not suitable for the construction of homogeneous porous structures by the anodization process. A homogeneous potential distribution at the electrode surface in a "symmetrical configuration" of a counter electrode to the $\mathrm{Au}$ electrode (Fig. 1) was essential for the fabrication of homogeneous porous structures. The effect of the applied potential on the porous structures at the Au surface has been previously reported. ${ }^{10,11}$ The FE-SEM image of the anodized Au electrode (Fig. S3) reveals threedimensional nanoporous structures on the surface. The nanoporous structure included stacked cylindrical components with macro/ mesopores between them. Overall, the nanoporous structures are 
A

(a)

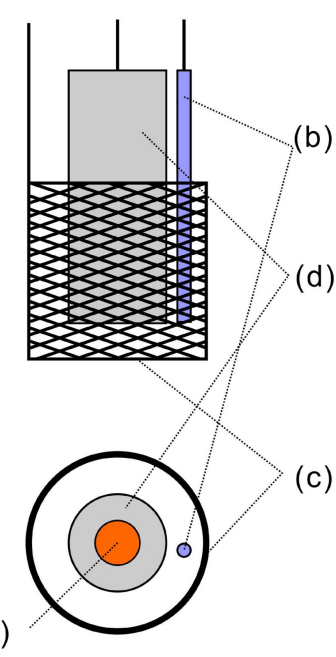

Figure 1. (A) Side and (B) bottom views of schematic of the electrode arrangement in the anodization of $\mathrm{Au}$ electrode; (a) $\mathrm{Au}$ working electrode, (b) reference electrode, (c) counter electrode, and (d) insulator of the working electrode.

similar to those reported previously. ${ }^{7}$ Considering the size of BOD, ${ }^{12}$ the pores seem to be sufficiently large to allow its penetration. The electrochemically active surface area of the electrode was evaluated based on the non-Faradaic current under anaerobic conditions. The ratio of the charging current of the anodized Au electrode to that of the planar electrode at $0.05 \mathrm{~V}\left(r_{\mathrm{r}}\right)$ has been used as a measure of the roughness factor of the anodized Au electrodes.

Figure 2A shows the rotating disk cyclic voltammograms (RDCVs) recorded at the BOD-adsorbed nanoporous Au electrodes anodized for $20 \mathrm{~s}\left(r_{\mathrm{r}}=6\right)$. The solid and dotted lines indicate the voltammograms recorded at $\mathrm{pH} 7.0$ under $\mathrm{O}_{2}$-saturated and anaerobic conditions, respectively. Catalytic reduction of $\mathrm{O}_{2}$ was observed at the BOD-adsorbed nanoporous Au electrode. A typical sigmoidal voltammogram has been recorded with a steady-state limiting current density of $-0.42 \mathrm{~mA} \mathrm{~cm}^{-2}$. Notably, such a sigmoidal voltammogram is rarely observed for the electroenzymatic reaction-controlled bioelectrocatalysis of BOD (cf. planar $\mathrm{Au}$ electrodes). The half-wave potential was $0.45 \mathrm{~V}$. This value agrees with the redox potential of the T1 site of BOD $(=0.460 \mathrm{~V}$ at $\mathrm{pH} 7.0^{13}$ ). Therefore, it can be concluded that a direct communication with $\mathrm{T} 1$, and not $\mathrm{T} 2 / 3$ cluster of $\mathrm{BOD}^{14}$ is predominant at the nanoporous $\mathrm{Au}$ electrodes.

The dashed line in Fig. $2 \mathrm{~A}$ shows the $\mathrm{O}_{2}$-reduction at the unmodified nanoporous $\mathrm{Au}$ electrode. The direct $\mathrm{O}_{2}$ reduction current at the nanoporous Au electrode ${ }^{17}$ was negligibly smaller than the catalytic reduction current of $\mathrm{O}_{2}$ at the BOD-modified electrodes.

The diffusion-limited current density under the RDCV conditions $\left(-8.4 \mathrm{~mA} \mathrm{~cm}^{-2}\right),{ }^{15}$ implies that the steady-state current is mainly controlled by the electro-enzymatic reaction. The limiting catalytic reduction current density of the nanoporous Au electrode was found to be 300 times higher than that of the BOD-adsorbed planar $\mathrm{Au}$ electrode (Fig. S4). The increase in the catalytic current density at the nanoporous Au electrode was greater than what was expected from the observed increase in the electrochemically active surface area. The result indicates that the DET-type bioelectrocatalysis of BOD is facilitated by an increase in not only the adsorbed amount of BOD, but also the population of the suitable orientation of adsorbed BOD.

The orientation of the adsorbed BOD was analyzed by the model of a randomly oriented spherical enzyme at a flat surface (details are shown in SI). ${ }^{16}$ There was no observable difference in the dispersion
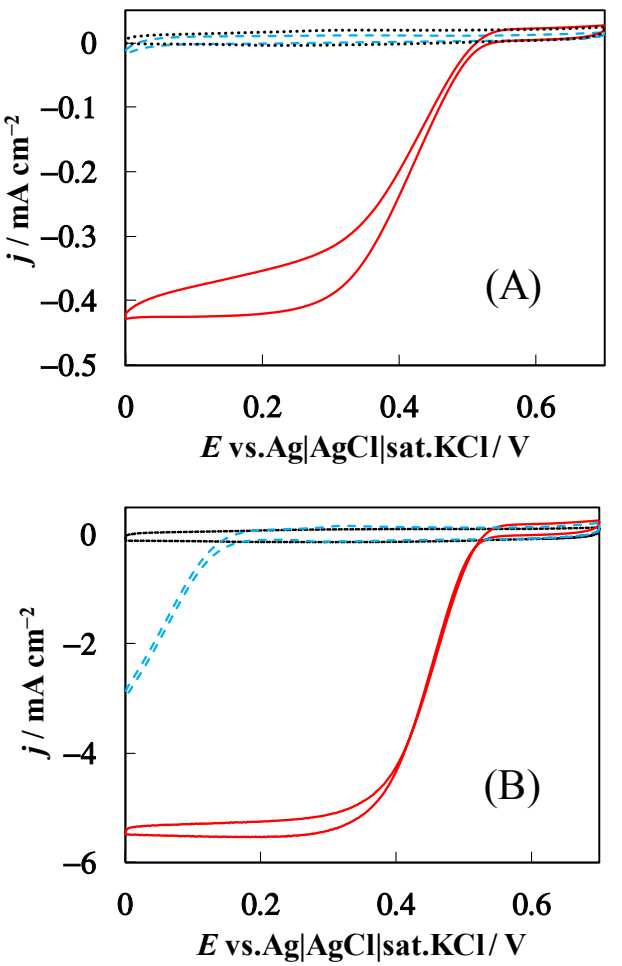

Figure 2. RDCVs recorded at the BOD-modified porous $\mathrm{Au}$ electrodes prepared with the anodization for (A) $20 \mathrm{~s}$ and (B) $450 \mathrm{~s}$. The solid and dotted lines indicate the RDCVs recorded under $\mathrm{O}_{2}$ saturated and Ar-saturated conditions, respectively. Dashed lines indicate the RDCVs recorded by the BOD-unmodified porous $\mathrm{Au}$ electrodes under $\mathrm{O}_{2}$-saturated conditions. The rotating speed and the scan rate were $4000 \mathrm{rpm}$ and $10 \mathrm{mV} \mathrm{s}^{-1}$, respectively.

in the orientation of BOD $(\Delta d)$ between the nanoporous and the planar $\mathrm{Au}$ electrodes. In contrast, assuming that the value of $k_{\mathrm{c}}$ remains constant, the effective amount of BOD at the nanoporous $\mathrm{Au}$ electrode was evaluated to be 300 times larger than that at the planar Au electrode. The increase in the effective amount of BOD cannot be simply explained by an increase in the electrochemically active surface area. A possible explanation is that most of the adsorbed BOD molecules on the planar Au surface have inactive orientations for the DET-type bioelectrocatalysis. The population of the enzymes that can play the role of a good catalyst increased drastically at the nanoporous $\mathrm{Au}$ surface owing to the curvature effect. Further study is required for the direct quantification of the adsorbed BOD.

The electrochemically active surface area of the nanoporous Au electrode increased with the anodization time. The solid line in Fig. 2B shows the RDCVs of $\mathrm{O}_{2}$ reduction on the nanoporous $\mathrm{Au}$ electrode prepared with an anodization time of $450 \mathrm{~s}\left(r_{\mathrm{r}}=200\right)$. In step with the growth of the surface area, the direct $\mathrm{O}_{2}$-reduction activity of the nanoporous $\mathrm{Au}$ electrode increased significantly at potentials lower than $0.2 \mathrm{~V}$ (dashed line in Fig. 2B); however, the contribution of the DET-type bioelectrocatalytic $\mathrm{O}_{2}$-reduction by BOD was still dominant. The DET-type $\mathrm{O}_{2}$ reduction reached the limited steady-state at potential values more negative than $0.3 \mathrm{~V}$ with the current density being approximately $-5.5 \mathrm{~mA} \mathrm{~cm}^{-2}$. This value is almost identical with the limiting steady-state current density of the BOD-immobilized nanoporous Au electrode prepared by a $6 \mathrm{~h}$ anodization in $0.5 \mathrm{M}$ glucose-containing phosphate buffer $\left(-5.1 \mathrm{~mA} \mathrm{~cm}^{-2}\right)^{9}$ and is close to the mass transfer limited value $\left(-8.4 \mathrm{~mA} \mathrm{~cm}^{-2}\right) \cdot{ }^{15}$ Additionally, the anodization in $\mathrm{KCl}$-containing solutions can be performed under quiescent conditions. Therefore, the present method for preparing nanoporous $\mathrm{Au}$ electrodes is 
superior to those previously proposed ${ }^{8,9}$ in terms of time, energy, and operability.

\section{Conclusions}

The anodization of $\mathrm{Au}$ in $\mathrm{KCl}$-containing buffer solutions under quiescent conditions was capable of producing effective scaffolds in a short time for the DET-type reaction of BOD. BOD immobilized on the nanoporous Au electrodes showed an increased population of the orientations favorable for the DET-type $\mathrm{O}_{2}$ reduction compared with the planar $\mathrm{Au}$ electrode. The $\mathrm{O}_{2}$ reduction current reached a value close to that limited by the $\mathrm{O}_{2}$ mass-transfer at potentials lower than $0.3 \mathrm{~V}$ despite neutral conditions.

\section{Supporting Information}

The Supporting Information is available on the website at DOI: https://doi.org/10.5796/electrochemistry.20-00079.

\section{Acknowledgments}

This work was supported by Kyoto University Nano Technology Hub in "Nanotechnology Platform Project" sponsored by the Ministry of Education, Culture, Sports, Science and Technology (MEXT), Japan. We would like to thank Editage (www.editage.com) for English language editing.

\section{References}

1. A. A. Karyakin, Bioelectrochemistry, 88, 70 (2012)

2. N. Mano and L. Edembe, Biosens. Bioelectron., 50, 478 (2013).

3. S. Tsujimura, H. Tatsumi, J. Ogawa, S. Shimizu, K. Kano, and T. Ikeda, J. Electroanal. Chem., 496, 69 (2001).

4. A. Walcarius, S. D. Minteer, J. Wang, Y. Lin, and A. Merkoçi, J. Mater. Chem. B, 1, 4878 (2013).

5. Y. Sugimoto, Y. Kitazumi, O. Shirai, and K. Kano, Electrochemistry, 85, 82 (2017).

6. J. Bhattarai, D. Neupane, B. Nepal, V. Mikhaylov, A. Demchenko, and K. Stine, Nanomaterials, 8, 171 (2018).

7. Y. Deng, W. Huang, X. Chen, and Z. Li, Electrochem. Commun., 10, 810 (2008).

8. K. Sakai, Y. Kitazumi, O. Shirai, and K. Kano, Anal. Sci., 34, 1317 (2018).

9. Y. Takahashi, M. Wanibuchi, Y. Kitazumi, O. Shirai, and K. Kano, J. Electroanal. Chem., 843, 47 (2019).

10. Y. Xia, W. Huang, J. Zheng, Z. Niu, and Z. Li, Biosens. Bioelectron., 26, 3555 (2011).

11. M. Kim, H. Jeong, E. Lee, and J. Kim, Bull. Korean Chem. Soc., 36, 2337 (2015).

12. J. A. Cracknell, T. P. Mcnamara, E. D. Lowe, and C. F. Blanford, Dalton Trans., 40, 6668 (2011).

13. S. Tsujimura, A. Kuriyama, N. Fujieda, K. Kano, and T. Ikeda, Anal. Biochem., 337, 325 (2005)

14. M. Dagys, A. Laurynėnas, D. Ratautas, J. Kulys, R. Vidžiūnaitė, M. Talaikis, G. Niaura, L. Marcinkevičienė, R. Meškys, and S. Shleev, Energy Environ. Sci., 10, 498 (2017).

15. K. So, M. Onizuka, T. Komukai, Y. Kitazumi, O. Shirai, and K. Kano, Electrochim. Acta, 192, 133 (2016)

16. H.-Q. Xia, Y. Kitazumi, O. Shirai, and K. Kano, J. Electroanal. Chem., 763, 104 (2016).

17. P. Rodriguez and M. T. M. Koper, Phys. Chem. Chem. Phys., 16, 13583 (2014). 